OPEN ACCESS

Edited by:

Wenbo Luo,

Liaoning Normal University, China

Reviewed by:

Bin Song,

McLean Hospital, United States

Tifei Yuan,

Nanjing Normal University, China

*Correspondence:

Bing Jin Li

libingjin@jlu.edu.cn

Wei Yang

wyang2002@jlu.edu.cn

Received: 31 May 2017

Accepted: 16 August 2017 Published: 04 September 2017

Citation:

Liu W, Leng YS, Zou XH, Cheng ZQ, Yang $W$ and Li BJ (2017) Affective Processing in Non-invasive Brain Stimulation Over Prefrontal Cortex.

Front. Hum. Neurosci. 11:439. doi: 10.3389/fnhum.2017.00439

\section{Affective Processing in Non-invasive Brain Stimulation Over Prefrontal Cortex}

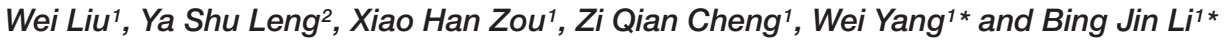 \\ 1 Jilin Provincial Key Laboratory on Molecular and Chemical Genetic, The Second Hospital of Jilin University, Changchun, \\ China, ${ }^{2}$ Department of Anesthesiology, The Third Hospital of Jilin University, Changchun, China
}

The prefrontal cortex (PFC) is the most frequently targeted brain region by noninvasive brain stimulation (NBS) studies. Non-invasively stimulating the PFC has been shown to both modulate affective processing and improve the clinical symptoms of several psychiatric disorders, such as depression and schizophrenia. The magnitude of the modulation depends on several factors, including the stimulation frequency, the number of stimulation sessions, and the specific sub-region of the PFC that is stimulated. Although some of the potential underlying mechanisms have been identified, the exact mechanisms that underlie these cognitive and affective changes remain unclear. The present review aims to summarize recent advances in the study of affective processing using NBS over the PFC. We will provide a theoretical framework for better understanding how affective processing changes are induced by NBS, with the goal of providing testable hypotheses for future studies.

Keywords: NBS, TMS, tDCS, tACS, affective processing, PFC

\section{INTRODUCTION}

Affective processing plays a significant role in survival and adaptation functions and often interacts with other cognitive processes such as memory, learning, decision making, motivation, and reactions to environmental circumstances (Davidson, 2002). Several biobehavioral scientists share the idea that there are two fundamental affective systems: those relating to positive and those relating to negative effects (Baker et al., 2014). Although the two systems rely on distinct neuroanatomical circuitry, increasing evidence suggests that the prefrontal cortex (PFC) and amygdala are involved in both systems (Pessoa and Adolphs, 2010; Li et al., 2017). Affective processing deficits are often a core factor in most psychiatric disorders such as depression, schizophrenia, phobia, anxiety disorder, bipolar disorder, and addiction. Non-invasive brain stimulation (NBS), which includes techniques such as transcranial magnetic stimulation (TMS) and transcranial direct/alternating current stimulation (tDCS/tACS), have been shown to be effective neuromodulating therapies for psychiatric disorders where impaired affective processing is a core feature, and have consequently shown great potential for future clinical applications. The PFC is the most frequently targeted region by NBS. To our knowledge, in contrast to the direct stimulation of the PFC, the effects of NBS on the amygdala are predominantly achieved through non-invasive stimulation of the PFC. 


\section{AFFECTIVE PROCESSING OF TMS OVER THE PFC}

Transcranial magnetic stimulation is developed on the basis of electromagnetic induction. The change of the extrinsic magnetic pulses on brain can produce an ionic current in the brain which can preferentially activate the pyramidal cells or at their axons and induce the change of neural excitability (Kobayashi and Pascual-Leone, 2003). Transcranial magnetic stimulation involves three modes: single pulse TMS, pair-pulse TMS, and repetitive TMS (rTMS). Repetitive TMS is the most mode in the therapy of depression and other psychiatric disorders. The Food and Drug Administration (FDA) has approved rTMS as an effective antidepressant treatment for medication-resistant patients with major depression. In clinical trials of psychiatric disorders, the PFC is the most studied brain regions with rTMS. The PFC is an important neural hub involved in mood and affective processing, in addition to playing an important role in the attentional control of affective information. The clinical effects of low-frequency $(1 \mathrm{~Hz})$ rTMS over the PFC are limited and significantly less than high-frequency $(>1 \mathrm{~Hz})$ rTMS (HFrTMS) over the PFC (Alonso et al., 2001; Cohen et al., 2004). Therefore, most of the studies of rTMS are based on HF-rTMS. High-frequency rTMS is known to induce a depolarization of the neurons in the PFC (Guse et al., 2010). Its application over the dorsolateral PFC (dlPFC) was shown to produce a greater cognitive improvement in patients with psychiatric/neurological diseases than in healthy participants (Guse et al., 2010). The exact underlying neural mechanisms responsible for this improvement remain unknown. Besides, the number of stimulation sessions is likely to play a role, as a single session of HF-rTMS applied over the dlPFC was not sufficient to produce significant mood changes in either normal volunteers or depressed patients (Baeken et al., 2008; Leyman et al., 2011).

\section{AFFECTIVE PROCESSING OF TMS OVER THE PFC IN DEPRESSION}

Affective processing of rTMS over the PFC has been shown in some affective disorders, such as depression, schizophrenia, and obsessive compulsive disorder. Depression is the most thoroughly studied in the clinical trials of rTMS. Repetitive TMS, as a typical mode of antidepressant treatment, has shown favorable effects on the depression. Although many studies have investigated the neural mechanisms of TMS over the PFC in depression, however, results are not always consistent and a clear picture has yet to emerge. High-frequency rTMS applied over the left dlPFC protects against the development of negative mood symptoms and increases the susceptibility to negative emotional stimuli (Möbius et al., 2016). High-frequency rTMS (10 Hz) over the left dIPFC of the depressed patients can improve the overall mood status, bias attentional processing toward negative information, and improve the Hamilton depression rating scale (Leyman et al., 2011). Although the first HF-rTMS stimulation session had no effect on the participants, there was a significant improvement following a 2-week treatment. However, no evidence showed the optimal treatment duration. The application of rTMS over the dIPFC also has effects on the cognitive control network and affects associated with cognitive control processes (Lantrip et al., 2017). However, measures of decision-making and impulse control were not affected by the application of rTMS over the left dIPFC, though measures of depression and anxiety both showed significant improvement (Tovar-Perdomo et al., 2017). This finding suggests that neurocognition may not be related to the psychopathological symptoms observed in these mood disorders. Furthermore, different rTMS stimulation frequency rates when applied over the dlPFC may have differential mood effects in depressed patients (Kimbrell et al., 1999; Speer et al., 2000; Speer et al., 2009). Although both low-frequency (1 Hz) and HF $(20 \mathrm{~Hz})$-rTMS have shown to produce positive results, depressed patients who display therapeutic improvements for one stimulation frequency may actually get worse when using other stimulation frequencies (Kimbrell et al., 1999). After 2 days of rTMS treatment, a widespread increase in cerebral blood flow was observed following high-frequency $(20 \mathrm{~Hz})$ stimulation, whereas low-frequency $(1 \mathrm{~Hz})$ stimulation produced a decrease in cerebral blood flow (Speer et al., 2009). Moreover, depressed patients with lower baseline metabolism levels showed a better response to high-frequency $(20 \mathrm{~Hz})$ stimulation, whereas those with higher baseline levels showed a better response to low-frequency $(1 \mathrm{~Hz})$ stimulation (Speer et al., 2000, 2009). In contrast, other studies have reported that both $5 \mathrm{~Hz}-\mathrm{rTMS}$ and $10 \mathrm{~Hz}$-rTMS may produce similar clinical effects on patients with major depressive disorder (MDD; Philip et al., 2015).

Several studies have demonstrated that important dlPFC hemispheric differences exist in MDD: the left dlPFC tends to be hypoactive, whereas the right dlPFC is generally hyperactive (Maeda et al., 2000; Mayberg, 2003; Phillips et al., 2003; Fahim et al., 2004; Bermpohl et al., 2006; Grimm et al., 2008; Lally et al., 2015). This finding suggests that the left and right dIPFC might serve distinct functions and be differently affected in MDD. The hypoactivity in the left dlPFC is related to emotional judgment and the modulation of positive emotional valence in MDD, whereas the hyperactivity in the right dlPFC is associated with attention to emotional judgment and depression severity in MDD (Grimm et al., 2008). Hyperactivation of the right dlPFC can also induce a consistent attentional bias toward negative and aversive memories.

The activation of the left dIPFC caused by rTMS treatment can improve the memory retrieval of positive affective memories, whereas symptoms of anxiety can be induced by the stimulation of the right dlPFC (Balconi and Ferrari, 2012). The application of rTMS over the left dlPFC produces an increased neuronal excitability in the left dlPFC, and recovered the normal responsivity to emotional judgment. It may be a mechanism of recovery in MDD. Besides, the application of rTMS over the left dIPFC can also increase brain activity in the left dlPFC and the anterior cingulate gyrus, whereas it has been shown to decrease brain activity in the left fusiform gyrus, the left cerebellum, and the right dlPFC (Cardoso et al., 2008). It was also shown that rTMS produced a similar clinical improvement in depressed patients when compared with a fluoxetine treatment. Another common clinical symptom observed in depressed patients is 
an inability to shift their attention focus, an impairment that is generally attributed to a dysfunction in the dlPFC. A single session of HF-rTMS over the left dlPFC can benefit task-switching performance and stabilize the patient's mood (Vanderhasselt et al., 2009). Although a single session of HFrTMS over the left dlPFC is insufficient to produce subjective mood changes, it can significantly affect the hypothalamicpituitary-adrenal (HPA)-axis by reducing salivary cortisol levels both immediately and 30 min after the stimulation (Baeken et al., 2009). These results indicate that changes involving the endocrine system may underlie the affective processing improvements.

The right dlPFC is related to symptoms of anxiety, depression, and panic. Chronic low-frequency rTMS over the right dlPFC showed a significant improvement in panic symptoms after 4 weeks of treatment, with the improvement peaking approximately after 8 weeks. However, the improvement of depressive symptoms required a longer treatment duration, where approximately only half the patients showed improvements after 8 weeks of treatment (Mantovani et al., 2013). The application of rTMS treatment to the right dlPFC can also increase subsequent event-related neural activity observed in bilateral, parietal, and temporal cortex; affect early stage affective visual processing; and enhance differential affective responses to fearful stimuli in individuals with anxiety (Zwanzger et al., 2014). Additionally, the right dlPFC may also be involved in the attentional processing of emotional information.

Multiple rTMS sessions applied to prefrontal regions may modulate the cortical excitability and improve the attentional control over emotional information. However, a single HF-rTMS session applied over the right dlPFC was shown to immediately impair the ability to inhibit negative information, whereas no significant changes were found when the left dlPFC was stimulated (Leyman et al., 2009). Such hemispheric stimulation differences were not observed for self-rated mood measures and emotionally induced facial expressions following dlPFC stimulation (Luo et al., 2010). Although both the left and right dlPFC play an important role in affective processing, sequential bilateral rTMS does not provide an added benefit compared with the application of HF-rTMS over the left dlPFC alone (Fitzgerald et al., 2012, 2016).

Besides the change of neural excitability, the alteration of various neurochemicals also contributes to neurobiological mechanisms of the antidepressant effect of rTMS. Repetitive TMS has been shown to change the endocrinological serum levels in the brain of healthy subjects (Evers et al., 2001). Infrathreshold stimulation only mildly decreased cortisol and thyroid stimulating hormone (TSH) serum levels in both the males and females. However, although this effect might stem from increased prolactin production in females, effects on other hormones were not observed. High-frequency rTMS $(25 \mathrm{~Hz})$ stimulations over the left dlPFC can decrease brain-derived neurotrophic factor (BDNF) serum levels in healthy men (Schaller et al., 2014). This effect can be further modulated by the smoking status of the male volunteers. It remains unknown whether sex is an additional modulating factor. However, the reported changes in BDNF levels have been inconsistent. Indeed, the findings of other studies have suggested that the use of acute rTMS over the PFC in healthy volunteers does not produce significant BDNF serum level changes (Lang et al., 2008). Moreover, rTMS applied over the dlPFC can also affect the cerebral glucose metabolism levels. In patients with medication-resistant depression, there are lower glucose metabolism that tends to be lower bilaterally in the dlPFC and in the anterior cingulum, whereas in contrast, several limbic and subcortical regions show increased glucose metabolism levels. After 2 weeks of rTMS treatment, however, glucose metabolism levels in the left middle temporal cortex and the fusiform gyrus significantly decreased in the patients who showed a better response; the general cerebral glucose metabolism pattern remained abnormal, however (Li et al., 2010). Finally, some animal studies and healthy human studies have shown that acute rTMS over the left dIPFC can transiently increase dopamine levels, whereas chronic rTMS does not produce the same effect (Kuroda et al., 2006).

The dorsomedial prefrontal cortex (dmPFC) is another important brain region that is affected by depression. Gray matter density of the dmPFC is reduced in depression, and the magnitude of this reduction correlates with the severity of depression (Koenigs et al., 2008; Koenigs and Grafman, 2009). Transcranial magnetic stimulation applied over the dmPFC can significantly improve the severity of clinical symptoms of depression by increasing the connectivity between the dmPFC and the thalamus, and also between the subgenual cingulate cortex and the caudate nucleus (Salomons et al., 2014; Dunlop et al., 2015; Schulze et al., 2016).

\section{AFFECTIVE PROCESSING OF TMS OVER THE PFC IN OTHER DISORDERS}

Transcranial magnetic stimulation is also increasingly considered as a promising treatment for schizophrenia. Active HF-rTMS $(10 \mathrm{~Hz})$ applied to the left dlPFC can induce a significant improvement of the negative clinical symptoms of schizophrenia and associated emotional functioning (Prikryl et al., 2013). These effects may be attributed to both an increase in neuronal activity and an increase of neurotransmitters or receptors. Stimulation over the left dlPFC may also enhance the neural connectivity between the PFC and the amygdala. However, high-frequency bilateral rTMS did not result in any substantial benefit regarding the negative symptoms of schizophrenia (Fitzgerald et al., 2008). The mechanism remains unknown. Deep rTMS applied over bilateral PFC also can significantly reduce self-oriented anxiety during difficult and affective social situations in patients with autism spectrum disorder (Enticott et al., 2014). Furthermore, rTMS applied over the left dlPFC can improve impaired affective processing in patients with obsessive compulsive disorder (de Wit et al., 2015). Potential underlying mechanisms include a reduction in neural activity within the left dlPFC and an increase in connectivity between frontal areas and the amygdala. In addition, rTMS over PFC also significantly decreased drug cue craving in drug-addicted patients, including heroin and cocaine, and no significant adverse events were noted (Shen et al., 2016; Terraneo et al., 2016). 


\section{AFFECTIVE PROCESSING OF tDCS OVER THE PFC}

Transcranial direct current stimulation is another non-invasive brain neuromodulation technique that is receiving more attention within the research community. In tDCS, a constant and low intensity direct current $(1-2 \mathrm{~mA})$ is delivered to the brain area via electrodes on the scalp. Direct current can influence the frequency of neuronal spontaneous discharge. The frequency gets higher when the anode of tDCS closes to the soma and dendrite of neurons, while the cathode of tDCS makes it lower (Wagner et al., 2007). Besides, tDCS can only affect the active neurons.

Transcranial direct current stimulation may affect cortical neuronal activity in the dIPFC, and the tDCS polarity (cathodal or anodal) applied to the dlPFC modulates the performance of participants during a stimulus-response compatibility task (Morgan et al., 2014). Cathodal current inhibits cortical excitability, whereas anodal current enhances it (Da et al., 2013). Transcranial direct current stimulation applied over the dlPFC had no influence, however, on self-reported affective state measures in healthy individuals (Morgan et al., 2014). However, the result was not consistent with the other studies in healthy volunteers. Polarity of tDCS to the left dlPFC had shown converse results in affective processing. Although some studies found that anodal tDCS applied to the left dIPFC decreased negative mood valence both during and after stimulation in healthy participants, other studies reported the opposite finding (Boggio et al., 2009; Peña-Gómez et al., 2011; Maeoka et al., 2012). Cathodal tDCS applied to the left dlPFC has been shown to have no effects on negative mood (Peña-Gómez et al., 2011; Nitsche et al., 2012). Moreover, tDCS has been shown to produce different physiological effects when applied to the left compared with the right dlPFC. Anodal tDCS applied to the right dlPFC (or cathodal tDCS to the left dlPFC) can significantly regulate affective impulses, whereas the opposite stimulation conditions can promote risky decision-making behavior (Pripfl et al., 2013).

The effects of tDCS on depression, schizophrenia, substance use disorders, obsessive compulsive disorder, generalized anxiety disorder, and anorexia nervosa have been extensively studied (Khedr et al., 2014; Kekic et al., 2016; Lefaucheur et al., 2017). Although some studies have reported its effectiveness for psychiatric disorders, generalizations should be made with caution given that the tDCS technique is still in its infancy compared to other brain stimulation techniques.

\section{AFFECTIVE PROCESSING OF tDCS OVER THE PFC IN DEPRESSION}

Transcranial direct current stimulation has been demonstrated as an effective, relatively safe and convenient treatment option for depression, which has been the most extensively studied clinical condition with this neuromodulation technique. The most studied brain region with tDCS in depression patients is the dlPFC, which plays a significant role in modulating emotions and motor cortex excitability. Cathodal stimulation current inhibits cortical excitability, whereas anodal current enhances it (Da et al., 2013; Brandão et al., 2015). The European Chapter of the International Federation of Clinical Neurophysiology produced guidelines that recommended the application of anodal tDCS over the left dIPFC (with a right orbitofrontal cathode) as a treatment option for patients with a MDD with (C level recommendation) and without drug resistance (B level recommendation) (Lefaucheur et al., 2017).

There are two neural networks involved in the neurobiology of depression. The first network underlies "hot" cognitive processes, is implicated in affective and reward processing, and is subserved by the limbic structures and the ventral PFC. The second network underlies "cold" cognitive processes; is related to non-emotional cognitive processing; and is subserved by the dorsal anterior cingulate cortex, the hippocampus, and the dlPFC (Drevets et al., 2008; Roiser and Sahakian, 2013; Nord et al., 2017). Cognitive deficits and executive dysfunctions are signature characteristics of major depression, and have been associated with the emotional dysregulation observed depression patients (Salehinejad et al., 2017). Ten sessions of tDCS applied over the dlPFC can significantly improve both the depressive symptoms and cognitive functions-such as working memory and attention-that are affected by depression. Working memory deficits are a common symptom associated with depression and other psychological conditions, such as schizophrenia. Working memory deficits can also be observed in healthy individuals exposed to situations producing acute stress. The application of anodal tDCS over the dlPFC can improve stress-induced working memory deficits in depression patients (Bogdanov and Schwabe, 2016). Furthermore, tDCS can enhance the improvement of working memory induced by neurocognitive training in depression patients (Vanderhasselt et al., 2015). Transcranial direct current stimulation can also modulate negative attentional bias measures in as little as a single tDCS session (Brunoni et al., 2014). The combined treatment of cognitive control training and tDCS applied over the dlPFC has been shown to produce a better improvement for depressed patients compared with tDCS alone (Segrave et al., 2014).

Transcranial direct current stimulation can enhance neuronal activation in the PFC, dorsal hippocampus, ventral tegmental area, and nucleus accumbens (Peanlikhit et al., 2017). Transcranial direct current stimulation also enhances vagal activation, inhibits the HPA activity, and decreases the salivary cortisol levels (Brunoni et al., 2013b). Unlike TMS, tDCS may have no significant influence on the BDNF levels in depression patients (Palm et al., 2013).

A recent study indicated that anodal tDCS stimulation of the left dlPFC (or cathodal stimulation of the right tDCS) reduced vigilance levels in response to threat-related affective stimuli in healthy individuals (Ironside et al., 2016). The authors of this study also put forward the idea that the processing of threatrelated affective information was a sensitive evaluation criterion to determine treatment effectiveness in clinical settings (Ironside et al., 2016). Finally, it has also been shown that anodal tDCS reduces cortisol levels and enhances vagal activity, both of which are involved in stress regulation (Brunoni et al., 2013b). 
Although there is no mechanism to reveal the interactions between tDCS with antipsychotics and nonbenzodiazepine anticonvulsants in depressed patients, some antipsychotics, such as SNRIs, SSRIs, and tricyclics, can significantly enhance the improvement of tDCS (Brunoni et al., 2013a). However, benzodiazepine can reduce the improvement associated with tDCS (Brunoni et al., 2013a). The use of a tDCS treatment after the administration of lorazepam (a GABAergic agonist) can initially reduce cortical excitability only to later increase excitability (Nitsche et al., 2004). Anodal stimulation has been shown to decrease local gamma-aminobutyric acid (GABA) levels, whereas cathodal stimulation inhibits glutamatergic activity (Stagg et al., 2009). GABA/glutamate levels are also modulated by benzodiazepine, which may explain the interaction effects observed when combining tDCS with benzodiazepines (Hasan et al., 2012).

\section{AFFECTIVE PROCESSING OF tDCS OVER THE PFC IN SCHIZOPHRENIA}

Transcranial direct current stimulation applied to the PFC has been shown to be an effective and relatively safe therapeutic option for adults and children with schizophrenia (Mattai et al., 2011). Negative symptoms and cognitive dysfunction are important facets of schizophrenia and are under the regulation of the fronto-thalamic-parietal and frontal-striatal networks (Galderisi et al., 2015; Palm et al., 2016). The dlPFC is a central hub in both of these networks and is functionally impaired in schizophrenia, leading to important cognitive deficits (Galderisi et al., 2015; Sheffield et al., 2015). Negative symptoms include a flattening of the affect, alogia, avolitionapathy, anhedonia-asociality, and attention disorders (Palm et al., 2016). Furthermore, the currently available evidence suggests that negative symptoms and cognitive dysfunctions are both associated with the affective processing deficits observed in schizophrenia. The application of prefrontal tDCS combined with stable antipsychotic medication has been shown to improve negative symptoms in patients with severe schizophrenia (Palm et al., 2016).

Prefrontal tDCS has also been shown to improve working memory performance and affective identification performance in patients with schizophrenia, and to enhance connectivity between brain networks in healthy individuals as measured by resting-state functional connectivity magnetic resonance imaging (fcMRI) (Rassovsky et al., 2015; Orlov et al., 2017). Auditory verbal hallucinations constitute another important clinical symptom in schizophrenia and are believed to be related to abnormal hyperactivity in the left temporoparietal junction, in addition to abnormal connectivity between frontal and temporal brain areas. The application of tDCS over the left dlPFC can significantly reduce the occurrence of auditory verbal hallucinations through the regulation of resting-state functional connectivity between brain areas in the related network (Mulquiney et al., 2011; Andrade, 2013; Mondino et al., 2016).

\section{AFFECTIVE PROCESSING OF tDCS OVER THE PFC IN DRUG DEPENDENCE AND BULIMIA NERVOSA}

The PFC also plays a critical role in developing addictions, particularly those related to drug use and smoking. Drug dependence has been associated with a reduction of brain activity within the PFC, which is believed to be subsequent to structural and functional damage to the PFC (Goldstein and Volkow, 2002; $\mathrm{Da}$ et al., 2013). Cravings are associated with enhanced drug cuerelated prefrontal activation (Tapert et al., 2003; Wilson et al., 2004; McBride et al., 2006). The application of tDCS over the left dIPFC is reported to inhibit the neural activation within the PFC normally triggered by drug-related cues, and to reduce acute substance cravings in drug addicts (Da et al., 2013; Wang et al., 2016). Moreover, tDCS can improve the general mood status of drug addict patients (Da et al., 2013).

Bulimia nervosa is an abnormal and excessive food craving disorder that can lead to other health problems including obesity. Some studies have indicated that the application of tDCS over either the right or the left dlPFC can enhance inhibitory control while reducing food cravings and intake (Burgess et al., 2016; Ljubisavljevic et al., 2016; Macedo et al., 2016; Georgii et al., 2017; Kekic et al., 2017; Lowe et al., 2017). The specific underlying mechanisms may involve an increased regulation of the reward and decision-making neural networks (Fregni et al., 2008).

\section{AFFECTIVE PROCESSING OF tDCS OVER THE PFC IN OTHER DISORDERS}

Posttraumatic stress disorder produces deficient fear extinction mechanisms and is associated with an abnormal activation of key brain regions that are part of the fear extinction network, which includes the ventromedial PFC, amygdala, and hippocampus (Marin et al., 2014). Furthermore, the integrity of this network is related to symptom severity in posttraumatic stress disorder. Transcranial direct current stimulation has been shown to enhance the recall of positive memories that improve fear extinction mechanism in posttraumatic stress disorder (Penolazzi et al., 2010; Asthana et al., 2013; Marin et al., 2014).

Pain is a multidimensional experience involving cognitive, attentional, and affective processes. Pain is often associated with negative emotions such as unpleasantness or anxiety, which can, in turn, modulate the pain experience. The application of tDCS over the left dlPFC can alleviate affective pain by increasing the neural activity in the left dlPFC, and also by down-regulating pain inhibitory systems (Maeoka et al., 2012). This mechanism may rely on a distinct and independent pathway from those regulating the somatosensory perception of pain (Boggio et al., 2009).

\section{AFFECTIVE PROCESSING IN tACS OVER THE PFC}

There are only a few studies that have investigated the effects of tACS on affective processing. These studies focused primarily 
on improving working memory functions in both healthy individuals and patients with schizophrenia (Meiron and Lavidor, 2014; Hoy et al., 2016). It has also been suggested that the bi-frontal application of tACS might improve functional connectivity between the different prefrontal regulatory components of working memory in humans. Furthermore, tACS applied to the left dlPFC can entrain endogenous cortical oscillations and produce working memory improvements in schizophrenia patients similar to those observed following tDCS.

\section{DISCUSSION}

Affective processing plays a significant role in survival and adaptation functions. Disturbance of affective processing is a core factor for most psychiatric disorders, such as, anxiety, depression, schizophrenia, phobia, and addiction. Although NBS studies have provided tantalizing symptom improvement and affective processing in some psychiatric disorders, such as depression and schizophrenia, there are still lots of limits for clinical applications. As we reviewed, depression is the most thoroughly studied affective disorder in TMS and tDCS; however, there are no standardized treatment protocols in the clinical application of rTMS for depressived patients. Repetitive TMS over the left dlPFC can improve the overall mood status, bias attentional processing toward negative information, increase susceptibility to negative emotional stimuli, and improve the Hamilton depression rating scale in the patients with depression. Repetitive TMS applied over the right dIPFC produces significant improvement in panic and depressive symptoms. Repetitive TMS treatment on the right dlPFC can also affect early stage affective visual processing, and enhance differential affective responses to fearful stimuli in individuals with anxiety. Transcranial direct current stimulation applied over the dlPFC can significantly improve the depressive symptom, cognitive function, such as working memory and attention, and stress-induced working memory deficits, and modulate the negative attentional bias in patients with depression. In depression, complicated pathogenesis, diversified pathogenic factor, manifold clinical symptoms, and individual differences make it difficult for the treatment of depression. Therefore, highquality multicenter randomized clinical trials are required to confirm the best optimal treatment for patients with depression, for instance, the frequency of rTMS, timing and course of the treatment, and evaluation of the therapeutic effect. In addition, some studies have indicated the changes of neural excitability and the alteration of various neurochemicals may be neurobiological mechanisms of the antidepressant effect of rTMS. Notwithstanding, there are also some potential

\section{REFERENCES}

Alonso, P., Pujol, J., Cardoner, N., Benlloch, L., Deus, J., Menchón, J. M., et al. (2001). Right prefrontal repetitive transcranial magnetic stimulation in obsessive-compulsive disorder: a double-blind, placebo-controlled study. Am. J. Psychiatry 158, 1143-1145. doi: 10.1176/appi.ajp.158.7.1143 mechanisms remaining to be revealed. In addition, the shortand long-term implications of NBS in patients with depression should get sufficient attention. Besides depression, other psychiatric disorders, for instance, schizophrenia, addiction, bulimia nervosa, and posttraumatic stress disorder, have exhibited favorable responses to NBS treatment. However, NBS also needs more evidence to verify the efficacy and explore physiological basis of affective processing in these psychiatric disorders.

\section{CONCLUSION}

The PFC is the most frequently studied region with NBS. The application of NBS over the PFC can significantly modulate affective processing and improve the clinical symptoms of several psychiatric disorders, such as depression and schizophrenia. The effects of TMS over the PFC on affective processing depend on the exact stimulation frequency, the number of consecutive stimulation sessions, and the targeted sub-region of the PFC. Although some potential mechanisms have been identified, the overall picture is yet to be complete. The application of TMS over the left dlPFC can increase brain activity in the left dlPFC and the anterior cingulate gyrus, whereas it also decreases brain activity measured in the left fusiform gyrus, the left cerebellum, and the right dlPFC. Transcranial magnetic stimulation applied to the right dlPFC can increase event-related neural activity bilaterally in the parietal and temporal cortex, increase early stage affective visual processing, and enhance differential affective responses to fearful stimuli. Transcranial direct current stimulation can enhance neuronal activation in the PFC, dorsal hippocampus, ventral tegmental area, and nucleus accumbens. However, the exact mechanisms underlying the tDCS effects remain unclear. As more and more studies investigate the effects and underlying mechanisms of NBS, its clinical applications should continue to be scrutinized.

\section{AUTHOR CONTRIBUTIONS}

$\mathrm{WL}, \mathrm{YL}, \mathrm{XZ}, \mathrm{ZC}$, and BL wrote the manuscript. WY and BL provided the critical revisions. All authors approved the final version of the manuscript for submission.

\section{FUNDING}

This work was supported by the National Natural Science Foundation of China (Grant Nos. 31571126 and 31300850) and the Graduate Innovation Fund of Jilin University.

Andrade, C. (2013). Transcranial direct current stimulation for refractory auditory hallucinations in schizophrenia. J. Clin. Psychiatry 74:e1054-8. doi: 10.4088/JCP. $13 \mathrm{f} 08826$

Asthana, M., Nueckel, K., Mühlberger, A., Neueder, D., Polak, T., Domschke, K., et al. (2013). Effects of transcranial direct current stimulation on consolidation of fear memory. Front. Psychiatry 4:107. doi: 10.3389/fpsyt.2013.00107 
Baeken, C., De Raedt, R., Leyman, L., Schiettecatte, J., Kaufman, L., Poppe, K., et al. (2009). The impact of one HF-rTMS session on mood and salivary cortisol in treatment resistant unipolar melancholic depressed patients. J. Affect. Disord. 113, 100-108. doi: 10.1016/j.jad.2008.05.008

Baeken, C., Leyman, L., De Raedt, R., Vanderhasselt, M. A., and D'haenen, H. (2008). Left and right high frequency repetitive transcranial magnetic stimulation of the dorsolateral prefrontal cortex does not affect mood in female volunteers. Clin. Neurophysiol. 119, 568-575. doi: 10.1016/j.clinph.2007. 11.044

Baker, J. T., Holmes, A. J., Masters, G. A., Yeo, B. T., Krienen, F., Buckner, R. L., et al. (2014). Disruption of cortical association networks in schizophrenia and psychotic bipolar disorder. JAMA Psychiatry 71, 109-118. doi: 10.1001/ jamapsychiatry.2013.3469

Balconi, M., and Ferrari, C. (2012). rTMS stimulation on left DLPFC affects emotional cue retrieval as a function of anxiety level and gender. Depress. Anxiety 29, 976-982. doi: 10.1002/da.21968

Bermpohl, F., Fregni, F., Boggio, P. S., Thut, G., Northoff, G., Otachi, P. T., et al. (2006). Effect of low-frequency transcranial magnetic stimulation on an affective go/no-go task in patients with major depression: role of stimulation site and depression severity. Psychiatry Res. 141, 1-13. doi: 10.1016/j.psychres. 2005.07.018

Bogdanov, M., and Schwabe, L. (2016). Transcranial stimulation of the dorsolateral prefrontal cortex prevents stress-induced working memory deficits. J. Neurosci. 36, 1429-1437. doi: 10.1523/JNEUROSCI.3687-15.2016

Boggio, P. S., Zaghi, S., and Fregni, F. (2009). Modulation of emotions associated with images of human pain using anodal transcranial direct current stimulation (tDCS). Neuropsychologia 47, 212-217. doi: 10.1016/j.neuropsychologia.2008. 07.022

Brandão, F. R. A., Baptista, A. F., Brandão, R. A., Meneses, F. M., Okeson, J., and de Sena, E. P. (2015). Analgesic effect of cathodal transcranial current stimulation over right dorsolateral prefrontal cortex in subjects with muscular temporomandibular disorders: study protocol for a randomized controlled trial. Trials 16:415. doi: 10.1186/s13063-015-0938-0

Brunoni, A. R., Ferrucci, R., Bortolomasi, M., Scelzo, E., Boggio, P. S., Fregni, F., et al. (2013a). Interactions between transcranial direct current stimulation (tDCS) and pharmacological interventions in the Major Depressive Episode: findings from a naturalistic study. Eur. Psychiatry 28, 356-361. doi: 10.1016/j. eurpsy.2012.09.001

Brunoni, A. R., Vanderhasselt, M. A., Boggio, P. S., Fregni, F., Dantas, E. M., Mill, J. G., et al. (2013b). Polarity- and valence-dependent effects of prefrontal transcranial direct current stimulation on heart rate variability and salivary cortisol. Psychoneuroendocrinology 38, 58-66. doi: 10.1016/j.psyneuen.2012. 04.020

Brunoni, A. R., Zanao, T. A., Vanderhasselt, M. A., Valiengo, L., de Oliveira, J. F., Boggio, P. S., et al. (2014). Enhancement of affective processing induced by bifrontal transcranial direct current stimulation in patients with major depression. Neuromodulation 17, 138-142. doi: 10.1111/ner.12080

Burgess, E. E., Sylvester, M. D., Morse, K. E., Amthor, F. R., Mrug, S., Lokken, K. L., et al. (2016). Effects of transcranial direct current stimulation (tDCS) on binge eating disorder. Int. J. Eat Disord. 49, 930-936. doi: 10.1002/eat.22554

Cardoso, E. F., Fregni, F., Martins, M. F., Boggio, P. S., Luis, M. M., Coracini, K., et al. (2008). rTMS treatment for depression in Parkinson's disease increases BOLD responses in the left prefrontal cortex. Int. J. Neuropsychopharmacol. 11, 173-183. doi: 10.1017/S1461145707007961

Cohen, H., Kaplan, Z., Kotler, M., Kouperman, I., Moisa, R., and Grisaru, N. (2004). Repetitive transcranial magnetic stimulation of the right dorsolateral prefrontal cortex in posttraumatic stress disorder: a double-blind, placebo-controlled study. Am. J. Psychiatry 161, 515-524. doi: 10.1176/appi.ajp.161.3.515

Da, S. M. C., Conti, C. L., Klauss, J., Alves, L. G., do, N. C. H. M., Fregni, F., et al. (2013). Behavioral effects of transcranial direct current stimulation (tDCS) induced dorsolateral prefrontal cortex plasticity in alcohol dependence. J. Physiol. Paris 107, 493-502. doi: 10.1016/j.jphysparis.2013.07.003

Davidson, R. J. (2002). Anxiety and affective style: role of prefrontal cortex and amygdala. Biol. Psychiatry 51, 68-80. doi: 10.1016/S0006-3223(01)01328-2

de Wit, S. J., van der Werf, Y. D., Mataix-Cols, D., Trujillo, J. P., van Oppen, P., Veltman, D. J., et al. (2015). Emotion regulation before and after transcranial magnetic stimulation in obsessive compulsive disorder. Psychol. Med. 45, 3059-3073. doi: 10.1017/S0033291715001026
Drevets, W. C., Price, J. L., and Furey, M. L. (2008). Brain structural and functional abnormalities in mood disorders: implications for neurocircuitry models of depression. Brain Struct. Funct. 213, 93-118. doi: 10.1007/s00429-0080189-x

Dunlop, K., Gaprielian, P., Blumberger, D., Daskalakis, Z. J., Kennedy, S. H., Giacobbe, P., et al. (2015). MRI-guided dmPFC-rTMS as a treatment for treatment-resistant major depressive disorder. J. Vis. Exp. 102:e53129. doi: 10.3791/53129

Enticott, P. G., Fitzgibbon, B. M., Kennedy, H. A., Arnold, S. L., Elliot, D., Peachey, A., et al. (2014). A double-blind, randomized trial of deep repetitive transcranial magnetic stimulation (rTMS) for autism spectrum disorder. Brain Stimul. 7, 206-211. doi: 10.1016/j.brs.2013. 10.004

Evers, S., Hengst, K., and Pecuch, P. W. (2001). The impact of repetitive transcranial magnetic stimulation on pituitary hormone levels and cortisol in healthy subjects. J. Affect. Disord. 66, 83-88. doi: 10.1016/S0165-0327(00) 00289-5

Fahim, C., Stip, E., Mancini-Marie, A., Mensour, B., Leroux, J. M., Beaudoin, G., et al. (2004). Abnormal prefrontal and anterior cingulate activation in major depressive disorder during episodic memory encoding of sad stimuli. Brain Cogn. 54, 161-163.

Fitzgerald, P. B., Herring, S., Hoy, K., McQueen, S., Segrave, R., Kulkarni, J., et al. (2008). A study of the effectiveness of bilateral transcranial magnetic stimulation in the treatment of the negative symptoms of schizophrenia. Brain Stimul. 1, 27-32. doi: 10.1016/j.brs.2007.08.001

Fitzgerald, P. B., Hoy, K. E., Elliot, D., McQueen, S., Wambeek, L. E., and Daskalakis, Z. J. (2016). A negative double-blind controlled trial of sequential bilateral rTMS in the treatment of bipolar depression. J. Affect. Disord. 198, 158-162. doi: 10.1016/j.jad.2016.03.052

Fitzgerald, P. B., Hoy, K. E., Herring, S. E., McQueen, S., Peachey, A. V., Segrave, R. A., et al. (2012). A double blind randomized trial of unilateral left and bilateral prefrontal cortex transcranial magnetic stimulation in treatment resistant major depression. J. Affect. Disord. 139, 193-198. doi: 10.1016/j.jad. 2012.02.017

Fregni, F., Orsati, F., Pedrosa, W., Fecteau, S., Tome, F. A., Nitsche, M. A., et al. (2008). Transcranial direct current stimulation of the prefrontal cortex modulates the desire for specific foods. Appetite 51, 34-41. doi: 10.1016/j.appet. 2007.09.016

Galderisi, S., Merlotti, E., and Mucci, A. (2015). Neurobiological background of negative symptoms. Eur. Arch. Psychiatry Clin. Neurosci. 265, 543-558. doi: 10.1007/s00406-015-0590-4

Georgii, C., Goldhofer, P., Meule, A., Richard, A., and Blechert, J. (2017). Food craving, food choice and consumption: the role of impulsivity and shamcontrolled tDCS stimulation of the right dlPFC. Physiol. Behav. 177, 20-26. doi: 10.1016/j.physbeh.2017.04.004

Goldstein, R. Z., and Volkow, N. D. (2002). Drug addiction and its underlying neurobiological basis: neuroimaging evidence for the involvement of the frontal cortex. Am. J. Psychiatry 159, 1642-1652. doi: 10.1176/appi.ajp.159. 10.1642

Grimm, S., Beck, J., Schuepbach, D., Hell, D., Boesiger, P., Bermpohl, F., et al. (2008). Imbalance between left and right dorsolateral prefrontal cortex in major depression is linked to negative emotional judgment: an fMRI study in severe major depressive disorder. Biol. Psychiatry 63, 369-376. doi: 10.1016/j.biopsych. 2007.05.033

Guse, B., Falkai, P., and Wobrock, T. (2010). Cognitive effects of high-frequency repetitive transcranial magnetic stimulation: a systematic review. J. Neural Transm. 117, 105-122. doi: 10.1007/s00702-009-0333-7

Hasan, A., Nitsche, M. A., Herrmann, M., Schneider-Axmann, T., Marshall, L., Gruber, O., et al. (2012). Impaired long-term depression in schizophrenia: a cathodal tDCS pilot study. Brain Stimul. 5, 475-483. doi: 10.1016/j.brs.2011.08.004

Hoy, K. E., Whitty, D., Bailey, N., and Fitzgerald, P. B. (2016). Preliminary investigation of the effects of $\gamma$-tACS on working memory in schizophrenia. J Neural Transm. 123, 1205-1212. doi: 10.1007/s00702-016-1554-1

Ironside, M., O’Shea, J., Cowen, P. J., and Harmer, C. J. (2016). Frontal cortex stimulation reduces vigilance to threat: implications for the treatment of depression and anxiety. Biol. Psychiatry 79, 823-830. doi: 10.1016/j.biopsych. 2015.06.012 
Kekic, M., Boysen, E., Campbell, I. C., and Schmidt, U. (2016). A systematic review of the clinical efficacy of transcranial direct current stimulation (tDCS) in psychiatric disorders. J. Psychiatr. Res. 74, 70-86. doi: 10.1016/j.jpsychires. 2015.12 .018

Kekic, M., McClelland, J., Bartholdy, S., Boysen, E., Musiat, P., Dalton, B., et al. (2017). Single-session transcranial direct current stimulation temporarily improves symptoms, mood, and self-regulatory control in bulimia nervosa: a randomised controlled trial. PLOS ONE 12:e0167606. doi: 10.1371/journal.pone. 0167606

Khedr, E. M., Elfetoh, N. A., Ali, A. M., and Noamany, M. (2014). Anodal transcranial direct current stimulation over the dorsolateral prefrontal cortex improves anorexia nervosa: a pilot study. Restor. Neurol. Neurosci. 32, 789-797. doi: 10.3233/RNN- 140392

Kimbrell, T. A., Little, J. T., Dunn, R. T., Frye, M. A., Greenberg, B. D., Wassermann, E. M., et al. (1999). Frequency dependence of antidepressant response to left prefrontal repetitive transcranial magnetic stimulation (rTMS) as a function of baseline cerebral glucose metabolism. Biol. Psychiatry 46, 1603-1613. doi: 10.1016/S0006-3223(99)00195-X

Kobayashi, M., and Pascual-Leone, A. (2003). Transcranial magnetic stimulation in neurology. Lancet Neurol. 2, 145-156. doi: 10.1016/S1474-4422(03)00321-1

Koenigs, M., and Grafman, J. (2009). Prefrontal asymmetry in depression? The long-term effect of unilateral brain lesions. Neurosci. Lett. 459, 88-90. doi: 10.1016/j.neulet.2009.04.063

Koenigs, M., Huey, E. D., Calamia, M., Raymont, V., Tranel, D., and Grafman, J. (2008). Distinct regions of prefrontal cortex mediate resistance and vulnerability to depression. J. Neurosci. 28, 12341-12348. doi: 10.1523/ JNEUROSCI.2324-08.2008

Kuroda, Y., Motohashi, N., Ito, H., Ito, S., Takano, A., Nishikawa, T., et al. (2006). Effects of repetitive transcranial magnetic stimulation on [11C]raclopride binding and cognitive function in patients with depression. J. Affect. Disord. 95, 35-42. doi: 10.1016/j.jad.2006.03.029

Lally, N., Nugent, A. C., Luckenbaugh, D. A., Niciu, M. J., Roiser, J. P., and Zarate, C. A. (2015). Neural correlates of change in major depressive disorder anhedonia following open-label ketamine. J. Psychopharmacol. 29, 596-607. doi: $10.1177 / 0269881114568041$

Lang, U. E., Hellweg, R., Gallinat, J., and Bajbouj, M. (2008). Acute prefrontal cortex transcranial magnetic stimulation in healthy volunteers: no effects on brain-derived neurotrophic factor (BDNF) concentrations in serum. J. Affect. Disord. 107, 255-258. doi: 10.1016/j.jad.2007.08.008

Lantrip, C., Gunning, F. M., Flashman, L., Roth, R. M., and Holtzheimer, P. E. (2017). Effects of transcranial magnetic stimulation on the cognitive control of emotion: potential antidepressant mechanisms. J. ECT 33, 73-80. doi: 10.1097/ YCT.0000000000000386

Lefaucheur, J. P., Antal, A., Ayache, S. S., Benninger, D. H., Brunelin, J., Cogiamanian, F., et al. (2017). Evidence-based guidelines on the therapeutic use of transcranial direct current stimulation (tDCS). Clin. Neurophysiol. 128, 56-92. doi: 10.1016/j.clinph.2016.10.087

Leyman, L., De Raedt, R., Vanderhasselt, M. A., and Baeken, C. (2009). Influence of high-frequency repetitive transcranial magnetic stimulation over the dorsolateral prefrontal cortex on the inhibition of emotional information in healthy volunteers. Psychol. Med. 39, 1019-1028. doi: 10.1017/ S0033291708004431

Leyman, L., De Raedt, R., Vanderhasselt, M. A., and Baeken, C. (2011). Effects of repetitive transcranial magnetic stimulation of the dorsolateral prefrontal cortex on the attentional processing of emotional information in major depression: a pilot study. Psychiatry Res. 185, 102-107. doi: 10.1016/j.psychres. 2009.04.008

Li, B., Ge, T., and Cui, R. (2017). Long-term plasticity in amygdala circuits: implication of CB1-dependent LTD in stress. Mol. Neurobiol. doi: 10.1007/ s12035-017-0643-y [Epub ahead of print].

Li, C. T., Wang, S. J., Hirvonen, J., Hsieh, J. C., Bai, Y. M., Hong, C. J., et al. (2010). Antidepressant mechanism of add-on repetitive transcranial magnetic stimulation in medication-resistant depression using cerebral glucose metabolism. J. Affect. Disord. 127, 219-229. doi: 10.1016/j.jad.2010.05.028

Ljubisavljevic, M., Maxood, K., Bjekic, J., Oommen, J., and Nagelkerke, N. (2016). Long-term effects of repeated prefrontal cortex transcranial direct current stimulation (tDCS) on food craving in normal and overweight young adults. Brain Stimul. 9, 826-833. doi: 10.1016/j.brs.2016.07.002
Lowe, C. J., Vincent, C., and Hall, P. A. (2017). Effects of noninvasive brain stimulation on food cravings and consumption: a meta-analytic review. Psychosom. Med. 79, 2-13. doi: 10.1097/PSY.00000000000 00368

Luo, W., Feng, W., He, W., Wang, N. Y., and Luo, Y. J. (2010). Three stages of facial expression processing: ERP study with rapid serial visual presentation. Neuroimage 49, 1857-1867. doi: 10.1016/j.neuroimage.2009.09.018

Macedo, I. C., de Oliveira, C., Vercelino, R., Souza, A., Laste, G., Medeiros, L. F., et al. (2016). Repeated transcranial direct current stimulation reduces food craving in Wistar rats. Appetite 103, 29-37. doi: 10.1016/j.appet.2016. 03.014

Maeda, F., Keenan, J. P., and Pascual-Leone, A. (2000). Interhemispheric asymmetry of motor cortical excitability in major depression as measured by transcranial magnetic stimulation. Br. J. Psychiatry 177, 169-173. doi: 10.1192/ bjp.177.2.169

Maeoka, H., Matsuo, A., Hiyamizu, M., Morioka, S., and Ando, H. (2012). Influence of transcranial direct current stimulation of the dorsolateral prefrontal cortex on pain related emotions: a study using electroencephalographic power spectrum analysis. Neurosci. Lett. 512, 12-16. doi: 10.1016/j.neulet.2012. 01.037

Mantovani, A., Aly, M., Dagan, Y., Allart, A., and Lisanby, S. H. (2013). Randomized sham controlled trial of repetitive transcranial magnetic stimulation to the dorsolateral prefrontal cortex for the treatment of panic disorder with comorbid major depression. J. Affect. Disord. 144, 153-159. doi: 10.1016/j.jad.2012.05.038

Marin, M. F., Camprodon, J. A., Dougherty, D. D., and Milad, M. R. (2014). Device-based brain stimulation to augment fear extinction: implications for PTSD treatment and beyond. Depress. Anxiety 31, 269-278. doi: 10.1002/da. 22252

Mattai, A., Miller, R., Weisinger, B., Greenstein, D., Bakalar, J., Tossell, J., et al. (2011). Tolerability of transcranial direct current stimulation in childhoodonset schizophrenia. Brain Stimul. 4, 275-280. doi: 10.1016/j.brs.2011. 01.001

Mayberg, H. S. (2003). Modulating dysfunctional limbic-cortical circuits in depression: towards development of brain-based algorithms for diagnosis and optimised treatment. Br. Med. Bull. 65, 193-207. doi: 10.1093/bmb/65. 1.193

McBride, D., Barrett, S. P., Kelly, J. T., Aw, A., and Dagher, A. (2006). Effects of expectancy and abstinence on the neural response to smoking cues in cigarette smokers: an fMRI study. Neuropsychopharmacology $\quad 31, \quad 2728-2738 . \quad$ doi: 10.1038/sj.npp. 1301075

Meiron, O., and Lavidor, M. (2014). Prefrontal oscillatory stimulation modulates access to cognitive control references in retrospective metacognitive commentary. Clin. Neurophysiol. 125, 77-82. doi: 10.1016/j.clinph.2013.06.013

Möbius, M., Lacomblé, L., Meyer, T., Schutter, D. J., Gielkens, T., Becker, E. S., et al. (2016). Repetitive transcranial magnetic stimulation modulates the impact of a negative mood induction. Soc. Cogn. Affect. Neurosci. 12, 526-533. doi: $10.1093 / \mathrm{scan} / \mathrm{nsw} 180$

Mondino, M., Jardri, R., Suaud-Chagny, M. F., Saoud, M., Poulet, E., and Brunelin, J. (2016). Effects of fronto-temporal transcranial direct current stimulation on auditory verbal hallucinations and resting-state functional connectivity of the left temporo-parietal junction in patients with schizophrenia. Schizophr. Bull. 42, 318-326.doi: 10.1093/schbul/ sbv114

Morgan, H. M., Davis, N. J., and Bracewell, R. M. (2014). Does transcranial direct current stimulation to prefrontal cortex affect mood and emotional memory retrieval in healthy individuals. PLoS ONE 9:e92162. doi: 10.1371/journal.pone. 0092162

Mulquiney, P. G., Hoy, K. E., Daskalakis, Z. J., and Fitzgerald, P. B. (2011). Improving working memory: exploring the effect of transcranial random noise stimulation and transcranial direct current stimulation on the dorsolateral prefrontal cortex. Clin. Neurophysiol. 122, 2384-2389. doi: 10.1016/j.clinph. 2011.05.009

Nitsche, M. A., Koschack, J., Pohlers, H., Hullemann, S., Paulus, W., and Happe, S. (2012). Effects of frontal transcranial direct current stimulation on emotional state and processing in healthy humans. Front. Psychiatry 3:58. doi: 10.3389/ fpsyt.2012.00058 
Nitsche, M. A., Liebetanz, D., Schlitterlau, A., Henschke, U., Fricke, K., Frommann, K., et al. (2004). GABAergic modulation of DC stimulationinduced motor cortex excitability shifts in humans. Eur. J. Neurosci. 19, 2720-2726. doi: 10.1111/j.0953-816X.2004.03398.x

Nord, C. L., Forster, S., Halahakoon, D. C., Penton-Voak, I. S., Munafò, M. R., and Roiser, J. P. (2017). Prefrontal cortex stimulation does not affect emotional bias, but may slow emotion identification. Soc. Cogn. Affect. Neurosci. 12, 839-847. doi: 10.1093/scan/nsx007

Orlov, N. D., Tracy, D. K., Joyce, D., Patel, S., Rodzinka-Pasko, J., Dolan, H., et al. (2017). Stimulating cognition in schizophrenia: a controlled pilot study of the effects of prefrontal transcranial direct current stimulation upon memory and learning. Brain Stimul. 10, 560-566. doi: 10.1016/j.brs.2016. 12.013

Palm, U., Fintescu, Z., Obermeier, M., Schiller, C., Reisinger, E., Keeser, D., et al. (2013). Serum levels of brain-derived neurotrophic factor are unchanged after transcranial direct current stimulation in treatmentresistant depression. J. Affect. Disord. 150, 659-663. doi: 10.1016/j.jad.2013. 03.015

Palm, U., Keeser, D., Hasan, A., Kupka, M. J., Blautzik, J., Sarubin, N., et al. (2016). Prefrontal transcranial direct current stimulation for treatment of schizophrenia with predominant negative symptoms: a double-blind, shamcontrolled proof-of-concept study. Schizophr. Bull. 42, 1253-1261. doi: 10.1093/ schbul/sbw041

Peanlikhit, T., Van Waes, V., Pedron, S., Risold, P. Y., Haffen, E., Etiévant, A., et al. (2017). The antidepressant-like effect of tDCS in mice: a behavioral and neurobiological characterization. Brain Stimul. 10, 748-756. doi: 10.1016/j.brs. 2017.03.012

Peña-Gómez, C., Vidal-Piñeiro, D., Clemente, I. C., Pascual-Leone, Á., and Bartrés-Faz, D. (2011). Down-regulation of negative emotional processing by transcranial direct current stimulation: effects of personality characteristics. PLoS ONE 6:e22812. doi: 10.1371/journal.pone.0022812

Penolazzi, B., Di, D. A., Marzoli, D., Mammarella, N., Fairfield, B., Franciotti, R., et al. (2010). Effects of transcranial direct current stimulation on episodic memory related to emotional visual stimuli. PLOS ONE 5:e10623. doi: 10.1371/ journal.pone.0010623

Pessoa, L., and Adolphs, R. (2010). Emotion processing and the amygdala: from a 'low road' to 'many roads' of evaluating biological significance. Nat. Rev. Neurosci. 11, 773-783. doi: 10.1038/nrn2920

Philip, N. S., Carpenter, S. L., Ridout, S. J., Sanchez, G., Albright, S. E., Tyrka, A. R., et al. (2015). $5 \mathrm{~Hz}$ repetitive transcranial magnetic stimulation to left prefrontal cortex for major depression. J. Affect. Disord. 186, 13-17. doi: 10.1016/j.jad. 2014.12.024

Phillips, M. L., Drevets, W. C., Rauch, S. L., and Lane, R. (2003). Neurobiology of emotion perception II: implications for major psychiatric disorders. Biol. Psychiatry 54, 515-528. doi: 10.1016/S0006-3223(03) 00171-9

Prikryl, R., Ustohal, L., Prikrylova, K. H., Kasparek, T., Venclikova, S., Vrzalova, M., et al. (2013). A detailed analysis of the effect of repetitive transcranial magnetic stimulation on negative symptoms of schizophrenia: a double-blind trial. Schizophr. Res. 149, 167-173. doi: 10.1016/j.schres.2013. 06.015

Pripfl, J., Neumann, R., Köhler, U., and Lamm, C. (2013). Effects of transcranial direct current stimulation on risky decision making are mediated by 'hot' and 'cold' decisions, personality, and hemisphere. Eur. J. Neurosci. 38, 3778-3785. doi: 10.1111/ejn.12375

Rassovsky, Y., Dunn, W., Wynn, J., Wu, A. D., Iacoboni, M., Hellemann, G., et al. (2015). The effect of transcranial direct current stimulation on social cognition in schizophrenia: a preliminary study. Schizophr. Res. 165, 171-174. doi: 10.1016/j.schres.2015.04.016

Roiser, J. P., and Sahakian, B. J. (2013). Hot and cold cognition in depression. CNS Spectr. 18, 139-149. doi: 10.1017/S10928529130 00072

Salehinejad, M. A., Ghanavai, E., Rostami, R., and Nejati, V. (2017). Cognitive control dysfunction in emotion dysregulation and psychopathology of major depression (MD): Evidence from transcranial brain stimulation of the dorsolateral prefrontal cortex (DLPFC). J. Affect. Disord. 210, 241-248. doi: 10.1016/j.jad.2016.12.036
Salomons, T. V., Dunlop, K., Kennedy, S. H., Flint, A., Geraci, J., Giacobbe, P., et al. (2014). Resting-state cortico-thalamic-striatal connectivity predicts response to dorsomedial prefrontal rTMS in major depressive disorder. Neuropsychopharmacology 39, 488-498. doi: 10.1038/npp.2013.222

Schaller, G., Sperling, W., Richter-Schmidinger, T., Mühle, C., Heberlein, A., Maihöfner, C., et al. (2014). Serial repetitive transcranial magnetic stimulation (rTMS) decreases BDNF serum levels in healthy male volunteers. J. Neural Transm. 121, 307-313. doi: 10.1007/s00702-013-1102-1

Schulze, L., Wheeler, S., McAndrews, M. P., Solomon, C. J., Giacobbe, P., and Downar, J. (2016). Cognitive safety of dorsomedial prefrontal repetitive transcranial magnetic stimulation in major depression. Eur. Neuropsychopharmacol. 26, 1213-1226. doi: 10.1016/j.euroneuro.2016. 04.004

Segrave, R. A., Arnold, S., Hoy, K., and Fitzgerald, P. B. (2014). Concurrent cognitive control training augments the antidepressant efficacy of tDCS: a pilot study. Brain Stimul. 7, 325-331. doi: 10.1016/j.brs.2013. 12.008

Sheffield, J. M., Repovs, G., Harms, M. P., Carter, C. S., Gold, J. M., MacDonald, A. W., et al. (2015). Fronto-parietal and cingulo-opercular network integrity and cognition in health and schizophrenia. Neuropsychologia 73, 82-93. doi: 10.1016/j.neuropsychologia.2015.05.006

Shen, Y., Cao, X., Tan, T., Shan, C., Wang, Y., Pan, J., et al. (2016). 10-Hz repetitive transcranial magnetic stimulation of the left dorsolateral prefrontal cortex reduces heroin cue craving in long-term addicts. Biol. Psychiatry 80, e13-e14. doi: 10.1016/j.biopsych.2016.02.006

Speer, A. M., Benson, B. E., Kimbrell, T. K., Wassermann, E. M., Willis, M. W., Herscovitch, P., et al. (2009). Opposite effects of high and low frequency rTMS on mood in depressed patients: relationship to baseline cerebral activity on PET. J. Affect. Disord. 115, 386-394. doi: 10.1016/j.jad.2008. 10.006

Speer, A. M., Kimbrell, T. A., Wassermann, E. M., Repella, J. D., Willis, M. W., Herscovitch, P., et al. (2000). Opposite effects of high and low frequency rTMS on regional brain activity in depressed patients. Biol. Psychiatry 48, 1133-1141. doi: 10.1016/S0006-3223(00)01065-9

Stagg, C. J., Best, J. G., Stephenson, M. C., O’Shea, J., Wylezinska, M., Kincses, Z. T., et al. (2009). Polarity-sensitive modulation of cortical neurotransmitters by transcranial stimulation. J. Neurosci. 29, 5202-5206. doi: 10.1523/JNEUROSCI. 4432-08.2009

Tapert, S. F., Cheung, E. H., Brown, G. G., Frank, L. R., Paulus, M. P., Schweinsburg, A. D., et al. (2003). Neural response to alcohol stimuli in adolescents with alcohol use disorder. Arch. Gen. Psychiatry 60, 727-735. doi: 10.1001/archpsyc. 60.7.727

Terraneo, A., Leggio, L., Saladini, M., Ermani, M., Bonci, A., and Gallimberti, L. (2016). Transcranial magnetic stimulation of dorsolateral prefrontal cortex reduces cocaine use: a pilot study. Eur. Neuropsychopharmacol. 26, 37-44. doi: 10.1016/j.euroneuro.2015.11.011

Tovar-Perdomo, S., McGirr, A., Van den Eynde, F., Rodrigues, D. S. N., and Berlim, M. T. (2017). High frequency repetitive transcranial magnetic stimulation treatment for major depression: dissociated effects on psychopathology and neurocognition. J. Affect. Disord. 217, 112-117.doi: 10.1016/j.jad.2017. 03.075

Vanderhasselt, M. A., De Raedt, R., Baeken, C., Leyman, L., and D'Haenen, H. (2009). A single session of rTMS over the left dorsolateral prefrontal cortex influences attentional control in depressed patients. World J. Biol. Psychiatry 10, 34-42. doi: 10.1080/156229707018 16514

Vanderhasselt, M. A., De Raedt, R., Namur, V., Lotufo, P. A., Bensenor, I. M., Boggio, P. S., et al. (2015). Transcranial electric stimulation and neurocognitive training in clinically depressed patients: a pilot study of the effects on rumination. Prog. Neuropsychopharmacol. Biol. Psychiatry 57, 93-99. doi: 10.1016/j.pnpbp.2014.09.015

Wagner, T., Fregni, F., Fecteau, S., Grodzinsky, A., Zahn, M., and Pascual-Leone, A. (2007). Transcranial direct current stimulation: a computer-based human model study. Neuroimage 35, 1113-1124. doi: 10.1016/j.neuroimage.2007.01.027

Wang, Y., Shen, Y., Cao, X., Shan, C., Pan, J., He, H., et al. (2016). Transcranial direct current stimulation of the frontal-parietal-temporal area 
attenuates cue-induced craving for heroin. J. Psychiatr. Res. 79, 1-3. doi: 10.1016/j.jpsychires.2016.04.001

Wilson, S. J., Sayette, M. A., and Fiez, J. A. (2004). Prefrontal responses to drug cues: a neurocognitive analysis. Nat. Neurosci. 7, 211-214.doi: 10.1038/ nn1200

Zwanzger, P., Steinberg, C., Rehbein, M. A., Bröckelmann, A. K., Dobel, C., Zavorotnyy, M., et al. (2014). Inhibitory repetitive transcranial magnetic stimulation (rTMS) of the dorsolateral prefrontal cortex modulates early affective processing. Neuroimage 101, 193-203. doi: 10.1016/j.neuroimage.2014. 07.003
Conflict of Interest Statement: The authors declare that the research was conducted in the absence of any commercial or financial relationships that could be construed as a potential conflict of interest.

Copyright $\odot 2017$ Liu, Leng, Zou, Cheng, Yang and Li. This is an open-access article distributed under the terms of the Creative Commons Attribution License (CC BY). The use, distribution or reproduction in other forums is permitted, provided the original author(s) or licensor are credited and that the original publication in this journal is cited, in accordance with accepted academic practice. No use, distribution or reproduction is permitted which does not comply with these terms. 